Stefan Kubica, Hagen Ringshausen,

Jörg Reiff-Stephan, Marius Schlingelhof (Hrsg.)

2. Automobil Symposium Wildau:

Tagungsband Technische Hochschule Wildau 2017
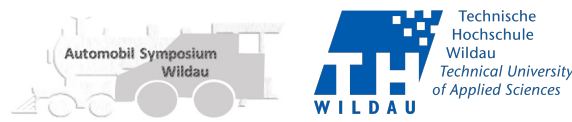

André Leschkee

Volkswagen $A G$

Florian Weinert

Volkswagen $A G$

\title{
NEUER SENSORANSATZ ZUR CRASHERKENNUNG IM PKW
}

\section{Einleitung}

Das Weißbuch der Europäischen Union setzt das Ziel, innerhalb der nächsten Dekade die Anzahl der im Verkehr getöteten Personen zu halbieren [Euro10]. Dieser Herausforderung als Teil der Vision Zero (dem Ziel, die Anzahl der Verkehrstoten auf null zu reduzieren) stellt sich die Automobilindustrie in den verschiedenen Entwicklungsbereichen. Ein an Bedeutung zunehmender Bestandteil der Maßnahmen zur Reduzierung der Verletzungsrisiken sind Systeme der aktiven Sicherheit. Aber auch die klassische passive Sicherheit mit ihren Schutzsystemen, wie Gurte und Airbags, bleibt weiterhin wichtig, da sich in absehbarer Zeit nicht alle Unfälle komplett vermeiden lassen werden.

Wesentlich bei der Betrachtung der heutigen Schutzsysteme ist, dass es sich um irreversible Bauteile handelt, die ihre optimale Schutzwirkung nur für wenige Millisekunden aufrechterhalten können. Aufgrund dieser Tatsache spielt der Auslösezeitpunkt im zeitlichen Ablauf eines Unfalles eine entscheidende Rolle für die erzielbare Schutzwirkung.

Zentraler Bestandteil heutiger Airbagsysteme ist das Airbagsteuergerät.
Während eines Unfalles werden in den hochkomplexen Algorithmen dieser Rechnereinheit u.a. die Schwere und die Richtung des vorliegenden Crashs bewertet. Auf Grundlage dieser Bewertung können dann die dazu passenden Auslösezeiten der im Fahrzeug eingebauten Rückhaltemittel, wie z.B. Airbags und Gurtstraffer, berechnet werden. Je nach Art des Unfalles müssen Auslöseentscheidungen Unfallgegner in einem Zeitintervall von ca. $10 \mathrm{~ms}$ - 50 ms nach Erstkontakt mit dem getroffen werden. Eine der wesentlichen Entscheidungsgrößen ist dabei die Fahrzeugverzögerung, die mit Hilfe von Beschleunigungssensoren ermittelt wird (Abbildung 1). [LeSG16].

Eine grundsätzliche Herausforderung dieses Messprinzips ist, dass die Fahrzeugverzögerung keine direkte Messgröße für einen Crash darstellt. So können Verzögerungen durch sogenannte Misuse-Events, wie z.B. Unfälle mit Wildtieren, das Durchfahren von großen Schlaglöchern oder das Anrutschen an einen Bordstein ebenfalls zu größeren, schlagartigen Fahrzeugverzögerungen führen.

Hier gilt es, im Rahmen des Möglichen eine korrekte Entscheidung zu treffen, um nicht unberechtigt und ohne Nutzen 


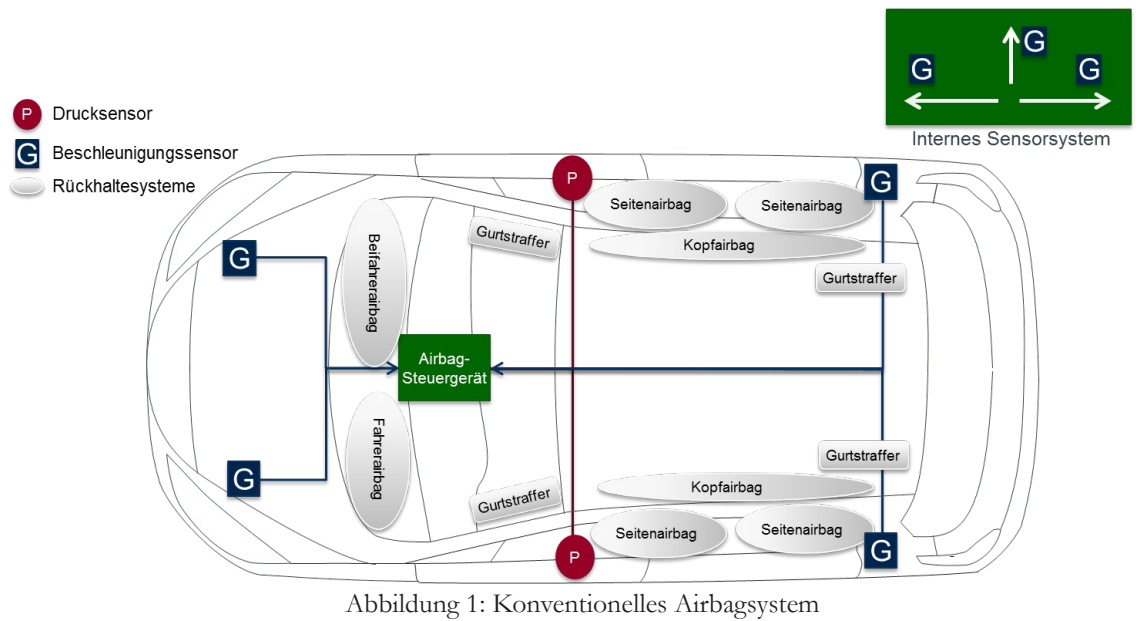

die Rückhaltemittel des Fahrzeugs auszulösen. Gerade in den extrem kurzen Zeitintervallen nach dem Beginn eines Unfalls unterscheiden sich die Verzögerungen durch ein Misuse-Event und die Verzögerungen durch einen Crash mit höherer Geschwindigkeit (z.B. OBD $64 \mathrm{~km} / \mathrm{h}$ aus Abbildung 2) mit einem anderen Fahrzeug nur marginal. Der Grund liegt in der physikalischen Struktur der „Unfallgegner“. Ein massiver,

harter Gegenstand, wie z.B. ein Parkplatzpoller oder eine Betonabtrennung, führt unmittelbar zur Verzögerung des auftreffenden Fahrzeugs. Eine Kollision mit einem anderen PKW führt aufgrund der weichen, Energie aufnehmenden „Knautschzone“ des Gegners zu Beginn des Unfalls nur zu geringen Verzögerungen (Abbildung 2), bis schließlich erst ab ca. $60 \mathrm{~ms}$ die harte Fahrzeugstruktur des Gegners wirksam wird.

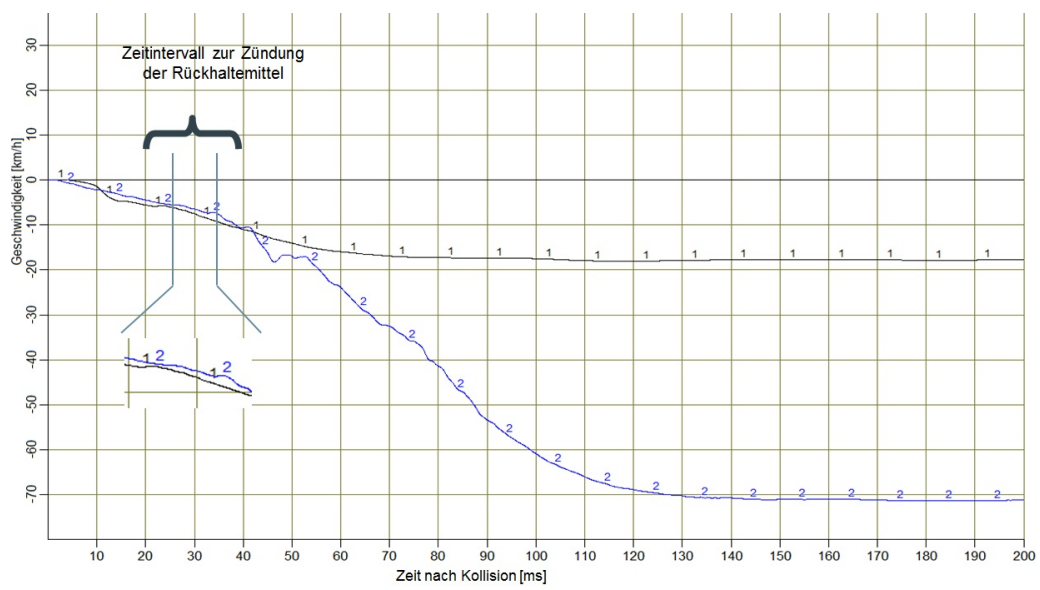

Abbildung 2: Signalverlauf RCAR $16 \mathrm{~km} / \mathrm{h}$ versus ODB $64 \mathrm{~km} / \mathrm{h}$ 


\begin{tabular}{|c|c|c|c|c|c|c|c|c|}
\hline Technology & NFC & $\begin{array}{l}\text { WLAN IEEE } \\
802.11 \mathrm{n}\end{array}$ & $\begin{array}{l}\text { WLAN IEEE } \\
802.11 p\end{array}$ & $\begin{array}{l}\text { WPAN } \\
802.15 .4\end{array}$ & NanoNet & SRD & RFID passive & div. \\
\hline frequency band & $13.56 \mathrm{MHz}$ & $2.4 \mathrm{GHz}$ & $5.85-5.925 \mathrm{GHz}$ & $2.4 \mathrm{GHz}$ & $2.4 \mathrm{GHz}$ & $868 \mathrm{MHz}$ & $868 \mathrm{MHz}$ & $\ldots$ \\
\hline transmit power & I & $100 \mathrm{mw}$ & $100 \mathrm{~mW}$ & $50 \mathrm{~mW}$ & $10 \mathrm{~mW}$ & $<500 \mathrm{~mW}$ & $100 \mathrm{~mW}$ & $\ldots$. \\
\hline bandwidth & I & $22 \mathrm{MHz}$ & $20 \mathrm{Mhz}$ & $1 \mathrm{MHz}$ & 80MHZ & $25 \mathrm{kHz}$ & $200 \mathrm{kHz}$ & $\cdots$ \\
\hline data rate & $<424 \mathrm{kBit} / \mathrm{s}$ & $150-600 \mathrm{MBit} / \mathrm{s}$ & 3-27 MBit/s & $<16 \mathrm{MBit} / \mathrm{s}$ & $<2 \mathrm{MBit} / \mathrm{s}$ & $<10 \mathrm{kBit} / \mathrm{s}$ & $<100 \mathrm{kBits} / \mathrm{s}$ & $\ldots$ \\
\hline $\begin{array}{l}\text { quantity of } \\
\text { connections }\end{array}$ & 1 & 30 & 400 & 16 & 2 & 10 & about 200 & $\ldots$ \\
\hline energy demand & low & high & mid & mid & mid & high & very low & $\ldots$ \\
\hline range & $<10 \mathrm{~cm}$ & $<70 m$ & $<1000 \mathrm{~m}$ & $<50 m$ & $60 \mathrm{~m}$ & $>1000 \mathrm{~m}$ & $<12 m$ & $\cdots$ \\
\hline latency & $<1 s$ & $<1$ s & $4-50 \mathrm{~ms}$ & $<1$ s & $<1 s$ & $<1$ s & $<5 \mathrm{~ms}$ & $\ldots$ \\
\hline disruptions & low & low & low & low & high & high & low & $\cdots$ \\
\hline cost sensor & low & high & high & high & mid & high & very low & $\ldots$ \\
\hline $\begin{array}{c}\text { effort for } \\
\text { integration }\end{array}$ & + & - & - & - & - & - & + & $\ldots$ \\
\hline overall & & & & & & & & \\
\hline
\end{tabular}

Abbildung 3: Vergleich verschiedener Funktechnologien

Darüber hinaus sind Beschleunigungssensoren nur sehr eingeschränkt in der Lage, Informationen zur genauen Richtung des Unfallgegners bereitzustellen.

Alle diese Herausforderungen sind heute auch auf Basis bekannter Sensorkonzepte lösbar, allerdings führt dies zum Verbau immer neuer zusätzlicher Sensoren an weiteren Positionen im Fahrzeug. Neben den Problemen, die durch den im Fahrzeug nur begrenzt verfügbaren Bauraum entstehen, müssen alle diese Sensoren verkabelt werden und lösen darüber hinaus die dargestellten physikalischen Probleme nicht grundsätzlich.

Im Rahmen dieses Papers wird ein grundlegend neuer Ansatz vorgestellt.

Das Ziel ist es, Intrusionen in der Fahrzeugstruktur direkt - zeitlich und örtlich - aufzulösen und damit eine dem Unfallverlauf proportionale Messgröße zu erzeugen.

Die verwendeten Sensoren sollen möglichst kabellos und damit einfach im Fahrzeug zu integrieren sein. Damit kann die Anzahl der Messstellen signifikant erhöht werden.

\section{Anforderungen an nicht leitungsgebundene Crashsensoren}

In einem übergreifenden Technologiescouting (Abbildung 3) sind verschiedenste Funktechnologien auf ihre grundsätzliche Eignung zum Einsatz als Crashsensor untersucht worden. Dabei sind die betrachteten Kriterien in zwei Kategorien einzuteilen.

Auf der einen Seite stehen anwendungsbezogene Kriterien wie die Integrationsfähigkeit ins Fahrzeug, auf der anderen Seite technologische Rahmenbedingungen wie Reichweite, Anzahl der möglichen gleichzeitigen Datenverbindungen, Datenrate und Energiebedarf. Im Gegensatz zu vielen anderen Anforderungen an drahtlose Kommunikation spielen hier die zeitlichen Aspekte, aufgrund des binnen weniger Millisekunden ablaufenden 
Fahrzeugcrashs, eine entscheidende Rolle. Deshalb ist eine möglichst kleine Latenz zwischen Sende- und Empfangseinheit einer der wesentlichen Faktoren.

Unter Berücksichtigung aller genannten Kriterien erfüllen passive RFID (Radio Frequency Identification) Tags die meisten der wesentlichen Anforderungen mit einem hohen Erfüllungsgrad. Insbesondere im Bereich Energiebedarf, Latenz und Integrationsfähigkeit zeigen sie das größte Potential aller betrachteten Technologien.

\section{Grundlagen der RFID- Technik}

Zum Aufbau einer Funkverbindung zur Datenübertragung sind RFID-Systeme zunächst vergleichbar mit anderen Funktechnologien. RFID Systeme bestehen grundsätzlich aus zwei Komponenten: dem Transponder (RFID-Tag), der im Wesentlichen Informationen bereitstellt, und der Auswerteeinheit. Der Transponder kann je nach Einsatzgebiet dabei auch mehrfach vorhanden sein. Diese Tags sind am Markt für eine Vielzahl von Anwendungen in diversen Bauformen verfügbar. Sie bestehen aus mindestens einem Mikrochip zur Datenverarbeitung und einer oder mehrerer Antennen zur Kommunikation. RFID-Systeme werden weiterhin in aktive und passive Systeme unterschieden. Aktive RFID-Systeme verfügen über eine Spannungsversorgung für die Transponder und die Auswerteeinheit und kommen daher für den geplanten Einsatz nicht infrage.

Passive RFID Tags haben darüber hinaus den Vorteil, dass sie keine eigene Energiequelle benötigen, sondern die zu ihrem Betrieb benötigte Energie aus dem Feld der Auswerteeinheit beziehen. Auch Auswerteeinheiten sind für eine Vielzahl von speziellen Anwendungsfällen am Markt zu finden. Sie bestehen mindestens aus einer Rechnereinheit zur Datenverarbeitung und einem Antennensystem zum Empfang der Nachrichten der Tags und zur Energieübertragung für deren Sendebetrieb, wie Abbildung 4 zeigt.

Der Betrieb erfolgt auf verschiedenen, ungeschützten Frequenzen, (z.B. $868 \mathrm{MHz}$, UHF-Band), den sich RFIDSysteme mit anderen Nutzern teilen müssen. Erzielbare Reichweiten liegen bei den am Markt befindlichen Systemen bei ca. 10m [Fink15].

Aus dem automotive-nahen Umfeld ist beispielsweise der Einsatz von RFID Technologie für logistische Zwecke bekannt. Durch den Einsatz von Tags auf Warengütern aller Art kann deren Weg durch die Produktionsanlagen jederzeit verfolgt werden. Die Anforderungen aus der Industrialisierung dieser Funktechnologie sind damit grundsätzlich schon gelöst.

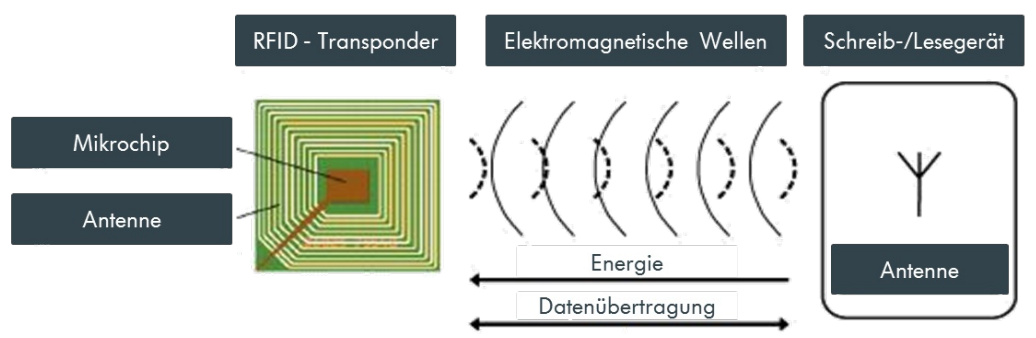

Abbildung 4: Schematische Darstellung der Datenübertragung passiver RFID-Tags [Krec05] 
Jedoch zieht der Einsatz für den in diesem Paper genannten Anwendungsfall eine Vielzahl von weiteren Anforderungen nach sich. Diese müssen untersucht werden und sind schließlich in einer Entwicklung eines RFID Crashsensortags massenmarkttauglich umzusetzen. Werden diese Anforderungen in Kategorien aufgeteilt, so bestehen Herausforderungen aus dem Einsatz im PKW-Umfeld. Dazu gehören z.B. mögliche Beeinflussungen oder Interferenzen aufgrund des hohen Metallanteils im Fahrzeug, Anforderungen an Lebensdauer, Temperatur und Feuchtigkeit und Anforderungen an speziell benötigte Bauformen zur Montage an Fahrzeugstrukturen.

Des Weiteren kommen noch spezifische Anforderungen aus dem Einsatz als Crashsensor hinzu. Beispielhaft zu nennen sind die sehr hohen Anforderungen an die Übertragungsgeschwindigkeit und die Anzahl gleichzeitig überwachter Tags in einem eng zusammen liegenden Umfeld.

Besonders an das Latenzverhalten im Detektionsprozess der Auswerteeinheit werden hohe Anforderungen gestellt. Eine Verarbeitung von mehreren
Dutzenden Tags im einstelligen Millisekunden Bereich ist erforderlich, um etwaige Crashsituationen rechtzeitig erfassen zu können.

\section{Sensorikkonzepte}

In Kapitel 1 wurde die Funktionsweise heutiger Airbagalgorithmen erläutert. Auf Basis von Fahrzeugverzögerungen, gemessen mit Beschleunigungssensoren, wird hier auf den vorliegenden Unfalltyp geschlossen. Airbagalgorithmen auf Basis von Informationen aus RFID Tags nutzen einen völlig anderen Erkennungsansatz. Hier soll direkt aus der Zerstörung der Fahrzeugstruktur bzw. der im Fahrzeug befindlichen Bauteile auf das vorliegende Crashereignis geschlossen werden (Abbildung 5).

In diesem Paper werden dazu zwei unterschiedliche Lösungsansätze vorgestellt.

\subsection{Bahnkurvenverfolgung}

In diesem Ansatz werden RFID Tags auf oder nahe der „Außenhaut" des Fahrzeuges verbaut. Die Tags sind über ihre ID und ihre bekannte Position im Koordinatensystem des Fahrzeugs eindeutig zu identifizieren und den

\section{Rückhaltesysteme}

RFID-Tag

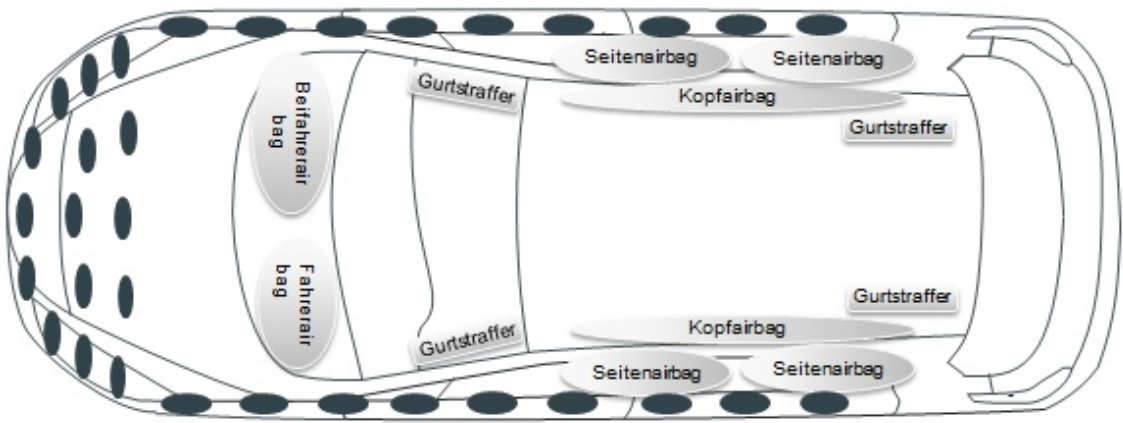

Abbildung 5: Prinzip Skizze: Verteilung RFID Tags im Fahrzeug 
vorher definierten Einbauorten im Fahrzeug zugeordnet. In einem Unfallszenario (z.B. einem Crash zwischen zwei Fahrzeugen) wird in der Regel die Fahrzeugstruktur an der Stoßstelle massiv verformt. Andere, nicht direkt durch den Stoß beaufschlagte Strukturen, bleiben dagegen unverformt. Dieses physikalische Verhalten wird nun in diesem Algorithmusansatz genutzt. Über das Auswertesteuergerät werden zyklisch alle Tags überwacht und ihre Position im Fahrzeugkoordinatensystem über Triangulations- bzw. Laufzeitverfahren bestimmt. Kommt es zu einer Kollision mit Intrusion (z.B. des rechten Kotflügels) bewegen sich die darauf befestigten Tags in Stoßrichtung und folgen der Verformung des Fahrzeugs. Durch Bestimmung der ID der sich räumlich verändernden Tags und ihrem jeweiligen absolut zurückgelegten Intrusionsweg, können direkt die Beschleunigung der getroffenen Bauteile und deren Ort bestimmt werden.

Da das typische Crashverhalten des jeweiligen Fahrzeuges in Applikationsversuchen bzw. Simulationen vorher bestimmt wurde, kann auf Basis dieser Informationen Crashart und -ort in einem realen Unfall genau bestimmt werden.

Dieses Verfahren bietet eine Vielzahl von Vorteilen. So ist die Zahl der pro Fahrzeug benötigten RFID Tags klein, das Messverfahren ist direkt proportional zur Fahrzeugzerstörung und der Integrationsaufwand im Fahrzeug ist zu vernachlässigen, da in der Regel in jedem Fahrzeugtyp gleiche oder ähnliche Positionen benutzt werden können. Auf der anderen Seite gibt es aber auch große Nachteile. Das benötigte Laufzeitverfahren ist extrem aufwendig und in einer realen Fahrzeugumgebung unter Berück- sichtigung der bestehenden Rahmenbedingungen nicht umsetzbar: ein typischer Crashtest des EuroNCAP, der sogenannte $64 \mathrm{~km} / \mathrm{h}$ ODB Versuch, führt zu einer Bewegung der Fahrzeugstruktur in den ersten $5 \mathrm{~ms}$ um ca. $0,9 \mathrm{~m}$. Die Laufzeit der Signale zwischen Tag und Auswerteeinheit laufen im idealen Fall mit Lichtgeschwindigkeit. Bei einem typischen Abstand zwischen dem Sender und dem Empfänger müsste hier eine Laufzeitdifferrenz von ca. 0,6ns gemessen werden. Unter Berücksichtigung von Bauteil- und Laufzeittoleranzen und zu erwartenden Störungen in der Fahrzeugumgebung ist eine so hochgenaue Messung technisch nicht oder nur mit unverhältnismäßig hohem Aufwand durchführbar. Trotz der großen Vorteile dieses Ansatzes, insbesondere der genauen Kenntnis über die Bahnkurven der im Crash beteiligten Fahrzeugstrukturen, ist dieser Ansatz nicht zielführend.

\subsection{Versagensmessung}

In diesem zweiten Ansatz werden RFID Tags im gesamten Fahrzeugaufbau verteilt.

Die Tags sind sowohl auf oder an den Fahrzeugkonturen, als auch in dahinter liegenden innenliegenden Fahrzeugbereichen an diversen dort befindlichen Bauteilen befestigt.

Die Tags sind hier ebenfalls über ihre ID und ihre bekannte Position im Koordinatensystem des Fahrzeugs eindeutig zu identifizieren und den vorher definierten Einbauorten im Fahrzeug zugeordnet. In einem Unfallszenario, wie z.B. einem Crash zwischen zwei Fahrzeugen, wird auch hier die Fahrzeugstruktur an der Stoßstelle massiv verformt. Andere, nicht direkt durch den Stoß beaufschlagte Strukturen, bleiben dagegen unverformt. 
Gemessen wird hier der Zeitpunkt der Zerstörung der an den getroffenen Strukturen befestigten RFID Tags. In einem typischen Fahrzeugcrash, bei dem z.B. schräg von vorne mit hoher Stoßgeschwindigkeit die Fahrzeugfront rechts getroffen wird, wird als erstes der Tag am Stoßfänger rechts, dann der Tag am Scheinwerfer rechts, dann am Montageträger rechts etc. getroffen. Aus dem Ausfallort der Tags kann auf die Stoßrichtung geschlossen werden. Über die Anzahl der nacheinander ausfallenden Tags und deren zeitliche Abfolge zueinander kann direkt auf die Crashart und die Crashstärke geschlossen werden(Abbildung 6).
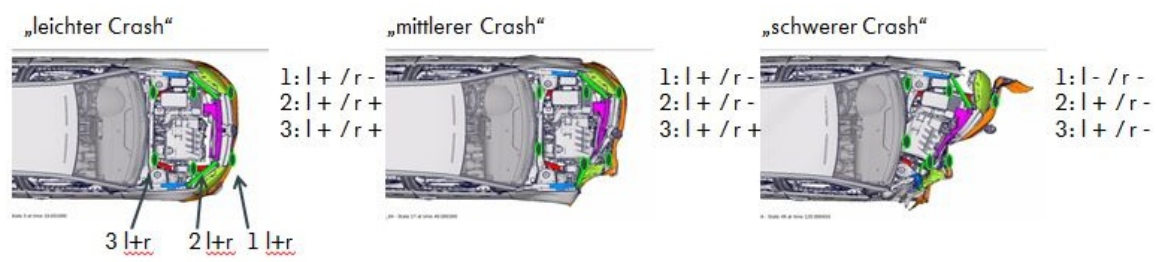

Abbildung 6: Versagensmessung
Die Ziffern 1 bis 3 kennzeichnen hier die Folge der Tags in Reihen, „"“ und „r“" kennzeichnen die Verbauposition auf der linken und rechten Seite. Dieses Verfahren ist technisch wesentlich einfacher umzusetzen, die Kosten für Antennensystem und Auswerteeinheit können wahrscheinlich in einem für die Massenanwendung akzeptablen Rahmen gehalten werden. Nachteilig ist, dass die Anzahl der benötigten Tags pro Fahrzeug deutlich größer wird und vor allem nebst Einbaupositionen für jedes Fahrzeug separat bestimmt werden müssen. Denn gerade der zeitliche Ablauf der Crashzerstörung im Fahrzeug von der Stoßstelle in den Fahrzeuginnenraum hinein ist stark vom jeweiligen Fahrzeugaufbau, der dabei verwendeten Stähle und der Anordnung der z.B. im
Motorraum befindlichen Bauteile abhängig.

\section{Algorithmus}

Die Basis für die generische Entwicklung und die fahrzeugspezifische Anpassung von Airbagalgorithmen sind Daten, die in (das Unfallgeschehen repräsentierenden) Crashtests in Laborumgebungen ermittelt werden.

Viele dieser Crashtests werden vom Gesetzgeber vorgegeben. Sie dienen in dem Fall nicht nur der Auslegung der Airbagalgorithmen, sondern auch zur Sicherstellung und zum Nachweis des Schutzpotentials des Fahrzeugs. Überprüft werden dabei sowohl das Verhalten der Fahrzeugstruktur, als auch die durch den Crash ausgeübten Belastungen auf die Insassen des Fahrzeuges. Diese Insassenwerte werden mit Hilfe speziell entwickelter Testpuppen, sogenannter Crash-Dummys, ermittelt. Zur Erfüllung der Vorgaben dürfen hierbei diverse Belastungswerte am Kopf, der Brust und weiteren Körperteilen nicht überschritten werden.

Sowohl die Art der durchzuführenden Crashs, als auch die Höhe der akzeptierten Belastungswerte, werden weltweit durch die lokalen Gesetzgeber jeweils länderspezifisch definiert. Dadurch ergibt sich eine Vielzahl von Anforderungen, die ein Automobil für eine Zulassung in den unterschiedlichen Märkten erfüllen muss.

Neben den Anforderungen durch die 
Gesetzgeber haben sich in den letzten Jahren weltweit Consumertestorganisationen gegründet, die mit verschärfen Anforderungen das Schutzpotential von Fahrzeugen im Kundeninteresse testen und mit sogenannten Sterneratings bewerten. Die bekanntesten Organisationen sind in Europa der EuroNCAP und in den USA die beiden Testorganisationen USNCAP und IIHS [Carh17].

Im vorherigen Kapitel wurde gezeigt, dass auf Basis der Zerstörung von RFID Tags im Ablauf eines Crashs Informationen über den Trefferpunkt am Fahrzeug, die Fahrzeugzerstörung und deren zeitlichen Abfolge gewonnen werden können.

Zur Erfüllung der diversen zu Beginn dieses Kapitels beschriebenen Anforderungen, müssen diese Informationen so ausgewertet werden, dass sie einen genauen Rückschluss auf das vorliegende Unfallereignis ermöglichen.

Dazu ist es notwendig, die RFID Tags im jeweiligen Fahrzeug so anzubringen, dass ausreichende Informationen vorliegen, um alle diese verschiedenen Crasharten eindeutig und rechtzeitig differenzieren zu können. Über Methoden der Mustererkennung, wie z.B. neuronalen Netzen, kann dann in realen Unfallgeschehen auf den vorliegenden Crashtyp und damit auf die benötigten Auslösezeiten der richtigen Rückhaltemittel geschlossen werden.

Beispielhaft wird im Folgenden das Vorgehen anhand der Lastfalltrennung zwischen zwei typischen Anforderungen aus dem europäischen Anforderungskontext gezeigt.

Bei beiden Crasharten handelt es sich um reine Frontcrashs. Auf der einen Seite handelt es sich um den RCAR $16 \mathrm{~km} / \mathrm{h}$ Test. Dies ist eine Anforderung aus dem Versicherungsumfeld, mit deren Hilfe die
Schadensklasse bei Fahrzeugversicherungen ermittelt wird. Aufgrund der geringen Differenzgeschwindigkeit und damit der kleinen Unfallschwere, ist hier für die Fahrzeuginsassen kein Rückhaltemittel als Schutzpotential notwendig. Es soll im Gegenteil zu 100 \% vermieden werden, dass Airbags oder Gurtstraffer auslösen, da das die Reparaturkosten und damit den sogenannten Schadenskorb der Versicherer unnötig erhöhen würde.

Auf der anderen Seite wird der schon aus Kapitel 1 bekannte $64 \mathrm{~km} / \mathrm{h}$ ODB Crash des EuroNCAP Testinstituts betrachtet. Hier liegt eine hohe Unfallschwere vor. Eine korrekte Auslösung der Rückhaltemittel ist damit essentiell notwendig.

Um beide Lastfälle differenzieren $\mathrm{zu}$ können, müssen die RFID Tags dazu ausreichende Informationen bereitstellen. In diesem auf Zerstörung der Tags basierenden Algorithmus bedeutet das, die Tags auf Fahrzeugstrukturen und Bauteilen zu befestigen, die in dem einen Fall zu 100\% sicher getroffen und in dem anderen Fall zu 100\% sicher nicht getroffen werden. Diese Einbauorte für die Tags müssen über Simulationsverfahren des Crashverlaufs für das spezifische Fahrzeug, in diesem Fall ein VW Golf, ermittelt werden.

Neben der sicheren Unterscheidung der Lastfälle kommt noch ein weiterer entscheidender Aspekt hinzu. Die benötigten Auslösezeiten für den 64 $\mathrm{km} / \mathrm{h}$ Test liegen bei ca. $30 \mathrm{~ms}$; eine sichere Trennung muss zwingend vor diesem Zeitpunkt möglich sein, da sonst eine zeitgerechte Auslösung der Rückhaltemittel nicht möglich ist. Eine Reihe von Simulationen mit unterschiedlichen Einbaupositionen führt $\mathrm{zu}$ der in der Abbildung beschriebenen Konstellation. 


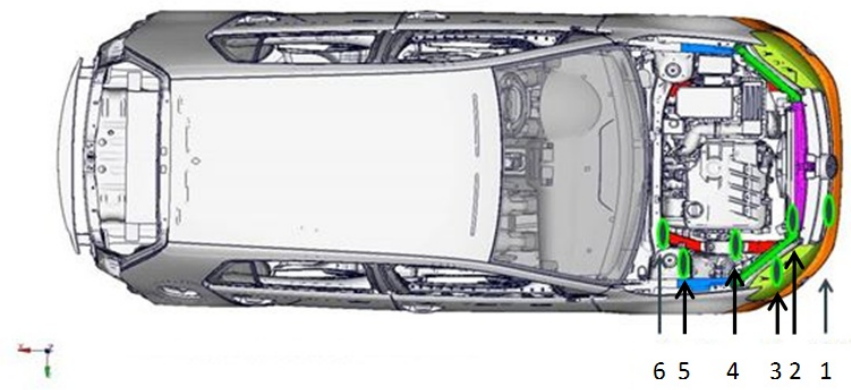

Abbildung 7: Positionierung RCAR $16 \mathrm{~km} / \mathrm{h}$ versus OBD $64 \mathrm{~km} / \mathrm{h}$

Je ein RFID Tag am Stoßfänger (1), Montageträger (2), Scheinwerfer (3), Motorblock (4), Federbeindom (5) und der Spritzwand (6).

Werden beide Crasharten in der Simulation betrachtet und die zeitlichen Abläufe der Tagzerstörung analysiert, ist $\mathrm{zu}$ beobachten, dass nach $20 \mathrm{~ms}$ im RCAR Test nur der Tag am Stoßfänger zerstört ist. Danach ist keine weitere Zerstörung zu sehen. Erst nach $40 \mathrm{~ms}$ wird noch der Tag am Montageträger getroffen. Dieses Verhalten (geringes Schadensbild, mit einem langsamen zeitlichen Fortschritt) ist typisch für einen Crash aus dem Lowspeed Bereich. Der $64 \mathrm{~km} / \mathrm{h}$ ODB Versuch verhält sich deutlich anders. Nach $20 \mathrm{~ms}$ sind bereits die ersten 3 Tags zerstört, $5 \mathrm{~ms}$ später kommt noch der vierte am Motorblock befestigte Tag dazu. Dieses Verhalten, hohes Schadensbild mit schnellem Zerstörungsfortschritt, ist typisch für einen Highspeedcrash. In diesem Beispiel ist damit eine sichere Trennung der beiden Lastfälle nach $20 \mathrm{~ms}$ bis $25 \mathrm{~ms}$ möglich und erlaubt damit die zeitgerechte Auslösung der benötigten Rückhaltemittel. Die beiden weiteren Tags am Federbeindom und der Schottwand sind zur Unterscheidung nicht mehr notwendig. Wenn nur diese beiden Lastfälle zu betrachten wären, könnten sie damit aus dem Sensorset entfernt werden.
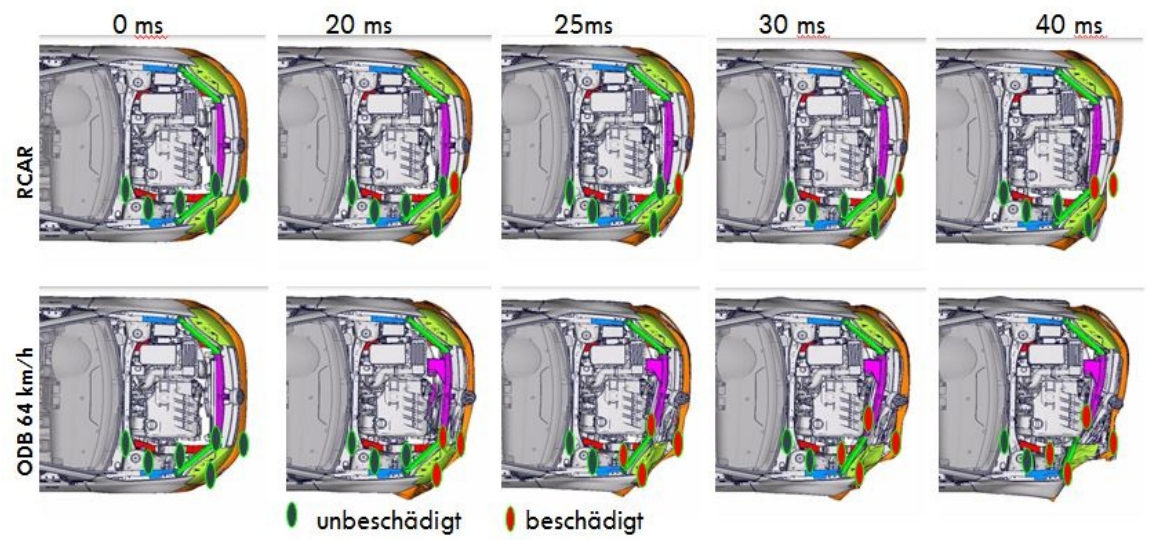

Abbildung 8: Positionierung RCAR $16 \mathrm{~km} / \mathrm{h}$ versus OBD $64 \mathrm{~km} / \mathrm{h}$ 
Wie am Anfang dieses Kapitels beschrieben, sind die Anforderungen in der Realität natürlich deutlich höher, so dass der hier dargestellte zweidimensionale Vergleich deutlich komplexer wird. Zur Ermittlung der notwendigen Sensorpositionen über die vielen Crasharten hinweg ist eine Vielzahl von Simulationsläufen notwendig.

Schwierig zu beherrschen sind hier vor allem Crasharten, die in ihrer Durchführung zum Teil nur über geringe Unterschiede in Geschwindigkeit, Aufbau der Crashbarriere oder der Trefferlage verfügen.

Grundsätzlich ist aus den bis jetzt bewerteten Lastfällen ersichtlich, dass eine Trennung prinzipiell immer durch die Zuhilfenahme weiterer Tags möglich ist.

\section{Zusammenfassung}

In diesem Paper wurde ein neuer Ansatz zur Crasherkennung in PKW Unfällen beschrieben. Heutige am Markt befindliche Sensorkonzepte bestehen in der Regel aus Beschleunigungssensoren. Dieses Messprinzip hat diverse Nachteile, die durch einen neuen Sensoransatz beseitigt werden sollen.

In einem Technologiescouting sind unterschiedliche drahtlose Funktechnologien auf ihre Tauglichkeit für einen Einsatz in einem Crashsensorkonzept untersucht worden. Passive RFID Tags erfüllen dabei die meisten Anforderungen sehr gut.

Zwei verschieden Konzepte zur Informationsgewinnung aus diesen Tags wurden untersucht und auf ihre Tauglichkeit bewertet. Zielführend zeigt sich hierbei ein Konzept, bei dem die zeitliche und örtliche Zerstörung von Tags im Verlauf des Fahrzeugcrashs gemessen und bewertet wird.
An einem konkreten Beispiel aus zwei Crasharten wurde ein darauf basierender Algorithmusansatz erläutert und die grundsätzliche Eignung von Technologie und Algorithmus gezeigt.

\section{Literaturverzeichnis}

[LeSG16]

Leschke, André, Seifert, Ulrich; Gonter, Mark: Kapitel 9 Fahrzeugsicherheit Vieweg Handbuch Kraftfahrzeugtechnik. Springer Verlag, Berlin, 2016

[Carh17]

carhs.training [Hrsg.]: Safety Companion 2017.

Eigenveröffentlichung der carhs.training $\mathrm{GmbH}$, Alzenau, 2017

[Fink15]

Finkenzeller, Klaus.: RFID-Handbuch. Hanser Verlag, München, 2015

[Euro10]

Europäische Kommission [Hrsg.]: Weißbuch zum Verkehr - Fahrplan zu einem einheitlichen europäischen Verkehrsraum - hin zu einem wettbewerbsorientierten und ressourcenschonenden Verkehrssystem. Amt für Veröffentlichungen der Europäischen Kommision, Luxemburg, 2010

[Krec05]

Krecak, Sinisa: Laufzeitmessung von RFID-Signalen zur ortsauflösenden Objektlokalisierung. Grin Verlag, München, 2005

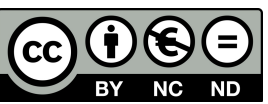

Dieser Beitrag ist unter der Creative-Commons-Lizenz CC BY-NC-ND lizensiert. 